\title{
The HMGB1 protein sensitizes colon carcinoma cells to cell death triggered by pro-apoptotic agents
}

\author{
CHENGCHENG CHRISTINE ZHANG ${ }^{1,2}$, GEORG GDYNIA $^{1,2}$, VOLKER EHEMANN $^{2}$ and WILFRIED ROTH $^{1,2}$ \\ ${ }^{1}$ Molecular Tumor Pathology, German Cancer Research Center (DKFZ), Im Neuenheimer Feld 280, D-69120 Heidelberg; \\ ${ }^{2}$ Institute of Pathology, University of Heidelberg, Im Neuenheimer Feld 224, D-69120 Heidelberg, Germany
}

Received September 21, 2014; Accepted November 6, 2014

DOI: $10.3892 /$ ijo.2014.2785

\begin{abstract}
The HMGB1 protein has multiple functions in tumor biology and can act both as a transcription factor and as a cytokine. HMGB1 is released during cell death, and in our previous studies we demonstrated that HMGB1 induces a distinct, necrosis-like cell death in glioblastoma. In epithelial malignant tumors such as colorectal cancer (CRC), the HMGB1-dependent effects show cross-talk with apoptotic signal transduction. Treatment of CRC cells with low concentrations of recombinant HMGB1 results in dose-dependent cytotoxicity which is morphologically characterized by the formation of giant mitochondria and does not share features of apoptosis. HMGB1-triggered cell death is associated with intracellular ROS release, and overexpression of Bcl-2 blocks both the increase of ROS as well as HMGB1-dependent cell death. Importantly, treatment with recombinant HMGB1 or overexpression of endogenous HMGB1 strongly sensitizes CRC cells to the cytotoxic activity of the pro-apoptotic death ligand TRAIL as well as the small molecule Bcl-2 family inhibitor ABT-737. Moreover, treatment of CRC cells with TRAIL or ABT-737 induces a release of endogenous HMGB1 into the extracellular space, and preincubation with glycyrrhizin, an HMGB1 inhibitor, significantly inhibits induction of cell death by TRAIL and ABT-737, suggesting that HMGB1 functionally contributes to the execution of cell death triggered by pro-apoptotic agents. Finally, we investigated the expression of HMGB1 in human CRC tumor samples and found that loss of HMGB1 expression is associated with a more aggressive phenotype and a more advanced stage of disease in patients with CRC. Altogether, our findings demonstrate a functional link between cytotoxic signaling cascades triggered by HMGB1 and pro-apoptotic agents leading to an HMGB1dependent sensitization to CRC cell death. Thus, a further
\end{abstract}

Correspondence to: Professor Wilfried Roth, Institute of Pathology, University of Heidelberg, Im Neuenheimer Feld 224, D-69120 Heidelberg, Germany

E-mail: wilfried.roth@med.uni-heidelberg.de

Key words: HMGB1, apoptosis, necrosis, colorectal cancer, synergy evaluation of recombinant HMGB1 as part of an experimental combination treatment of CRC seems warranted.

\section{Introduction}

High-mobility group box 1 (HMGB1) is a member of the 'high mobility group (HMG) nuclear proteins' (1). Structurally, HMGB1 contains two homologue DNA-binding elements, the N-terminal A-Box and the central B-Box, and a negatively loaded C-terminus (186-215) (2). Furthermore, HMGB1 has two nuclear localization sequences (3). In the human body, HMGB1 has a dual function since it can act both as a transcription factor and as a cytokine. As a transcription factor, HMGB1 is expressed in most of the eukaryotic cells. It binds to the minor groove of the DNA (4) and regulates the expression of different genes (1). As a cytokine, HMGB1 is released during inflammatory and infectious processes by activated macrophages, monocytes, dendritic cells and natural killer cells $(2,5,6)$. LPS (lipopolysaccharide) and interleukin-1 (IL-1) have stimulatory effects on the HMGB1 secretion (7). In addition, during necrosis nuclear HMGB1 dissociates from the chromatin and is released into the extracellular space after being acetylated, thereby acting as a 'danger signal' for neighboring cells (8). However, HMGB1 is not released by apoptotic cells in most cell types because of generalized underacetylation of histones and consecutive strong interactions between HMGB1 and chromatin (8). Nevertheless, in some cell types HMGB1 release occurs during late apoptosis, which might also be considered as secondary necrosis (9).

Extracellular HMGB1 binds to different receptors involved in innate immunity, such as RAGE, TLR2, TRL4 and TLR9, thereby triggering pleiotropic effects (10-12). HMGB1 promotes inflammatory processes (5), activates chemotaxis of cells, functions as a danger signal for other cells, and activates the immune system $(8,13,14)$. HMGB1 is also an important mediator in the pathogenesis of several diseases such as arthritis, sepsis and cancer $(2,14,15)$. In the context of cancer, both pro- and antitumoral effects of HMGB1 have been described. In our previous studies we demonstrated that HMGB1 induces a distinct, necrosis-like type of cell death in glioblastoma which is morphologically characterized by the formation of giant mitochondria (16). However, this novel type of cell death seems to be differentially regulated in various forms of cancer. In colorectal cancer (CRC) cells, HMGB1 
induces a specific cellular response not observed in other types of tumors. Specifically, HMGB1-dependent signaling intricately cross-talks with apoptotic signal transduction. In this study, we sought to characterize and identify the cellular mechanisms regulating the differential cell death signaling triggered by HMGB1 and pro-apoptotic agents. CRC is a disease with a poor prognosis for patients with metastasized disease, and in part this therapy resistance is caused by cellular resistance to apoptosis. Thus, agents that sensitize CRC cells to apoptotic cell death are potential candidates for novel therapeutic approaches for patients with CRC.

\section{Materials and methods}

Materials. rhHMGB1 and glycyrrhizin were purchased from Sigma-Aldrich (Deisenhofen, Germany). Soluble TRAIL was purchased from Biomol (Hamburg, Germany). ABT-737 was provided by Selleck Chemicals (Houston, TX, USA).

Cell culture and viability assays. Human cancer cell lines were purchased from the American Type Culture Collection (ATCC, Rockville, MD, USA), expanded and frozen in aliquots within four weeks. For experiments, the cells were thawed and cultured for no more than eight weeks. The human colorectal carcinoma cell lines SW480A and Hct116 were cultured in RPMI (Invitrogen, Carlsbad, CA, USA, \#21875-034) containing 10\% FCS (PAA, Piscataway, NJ, USA, A15-151) and 1\% penicillin/streptomycin (Invitrogen, \#15140-122). For acute cytotoxicity assays, $5 \times 10^{4}$ cells were plated in 96-well plates, adhered for $24 \mathrm{~h}$ and exposed to rhHMGB1, ABT-737 or TRAIL alone or in combination for 24 and 72 h. For inhibition of endogenous HMGB1 cells were treated with $1 \mathrm{mM}$ glycyrrhizin. The percentage of surviving cells was assessed by staining with crystal violet (17). After incubation the supernatant was removed and the cells were incubated in a $2 \%$ crystal violet solution (AcROS Organics, Geel, Belgium) in $20 \%$ methanol (Fluka, Munich, Germany) for $10 \mathrm{~min}$. The plates were washed in water and air-dried for $24 \mathrm{~h}$. Crystal violet was solubilized by the addition of a $0.1 \mathrm{M}$ sodium citrate buffer (Fluka) in 50\% ethanol. The absorption was measured at $550 \mathrm{~nm}$ using a microplate reader model 680 (Bio-Rad Instruments, Munich, Germany).

Electron microscopy. After treatment, cells were fixed with $2.3 \%$ glutaraldehyde in $50 \mathrm{mM}$ sodium cacodylate $(\mathrm{pH} 7.2)$ for $30 \mathrm{~min}$ at $4^{\circ} \mathrm{C}$, scraped from the plates and centrifuged at $200 \mathrm{x} \mathrm{g}$ for $10 \mathrm{~min}$ at $4^{\circ} \mathrm{C}$. The sediment was stained with $2 \%$ osmium tetroxide and $0.5 \%$ uranyl acetate. After dehydration and embedding in Epon (Shell, The Hague, The Netherlands), the probes were processed for ultrathin section. Micrographs were taken with a Zeiss EM-10A electron microscope at $80 \mathrm{kV}$ (Jena, Germany).

Immunohistochemistry. The human tissue samples were provided by the Tumor Tissue Bank of the National Center for Tumor diseases (NCT) in Heidelberg after approval by the ethics committee of the University of Heidelberg. The collection of tissue samples comprised 47 primary colorectal adenocarcinomas with pT3 $(n=44)$ or pT4 stage $(n=3)$ and the following features: $\mathrm{G} 2, \mathrm{n}=20 ; \mathrm{G} 3 / 4, \mathrm{n}=27$; $\mathrm{pN} 0, \mathrm{n}=23$; $\mathrm{pN} 1+$, $\mathrm{n}=24$. The tissue microarray slides were dewaxed and rehydrated using xylene (Merck, Darmstadt, Germany) and a series of graded alcohols. For antigen retrieval the slides were incubated with target retrieval solution (Dako REAL ${ }^{\mathrm{TM}}$ Proteinase K Diluent, Dako, Hamburg, Germany, \#S2031) in a steamer for $30 \mathrm{~min}$. Staining was performed by using an automated staining system (Techmate 500; DakoCytomation, Glostrup, Denmark) with anti-HMGB1 (Epitomics, CA, USA) 1:250 overnight and avidin-biotin-complex peroxidase technique using aminoethylcarbazole (Dako) for visualization the next day. Haemalaun (Merck) was used for counterstaining.

Immunoblot analysis. Colorectal carcinoma cells were rinsed with ice-cold PBS (Invitrogen, Groningen, The Netherlands) and harvested with a cell scraper in cold lysis buffer [120 mM NaCl, $50 \mathrm{mM}$ Tris- $\mathrm{HCl}$ (pH 8.0), $5 \mathrm{mM}$ EDTA, $0.5 \%$ Triton X-100 (Sigma-Aldrich)] containing $1 \mathrm{mM}$ phenylmethylsulfonylfluoride (Alexis, Lörrach, Germany), proteinase inhibitors (Roche, Mannheim, Germany, \#1697498) and phosphatase inhibitors (25 mM NaF, $200 \mu \mathrm{M} \mathrm{NaVO}_{3}$, $10 \mathrm{mM}$ NaPP) (Sigma-Aldrich). After 15-min incubation on ice, the lysates were centrifuged at $16,000 \mathrm{x}$ g for $20 \mathrm{~min}$. The total protein concentration of the lysates was measured using the Bradford-Assay (Bio-Rad, \#500-0006). Soluble protein (10-30 $\mu \mathrm{g}$ per lane) was separated on $10-15 \%$ polyacrylamide gels and blotted onto nitrocellulose membrane (Bio-Rad, \#162-0115) by standard procedures. The membranes were washed, incubated with primary antibody as indicated, washed again, and incubated with a secondary antibody (1:3,000, horseradish peroxidase-conjugated, Bio-Rad). Bound antibodies were visualized using an enhanced chemiluminescence (ECL) detection system [GE Health-care, (ORT) UK, \#RPN2109]. The membranes were incubated with various primary antibodies, including mouse anti-Bcl-2 (1:1,000, Santa Cruz, Heidelberg, Germany), rabbit anti-HMGB1 (1:1,000, Abcam, Cambridge, UK), mouse anti-Flag antibody (1:1,000, Sigma Chemical Co., St. Louis, MO, USA), mouse anticaspase 3 antibody (1:1,000, Imgenex, San Diego, CA, USA), mouse anti-cytochrome $c$ antibody (1:1,000, Abcam), mouse anti-cytochrome $c$ oxidase complex IV antibody $(1: 1,000$, Molecular Probes, Invitrogen, Karlsruhe, Germany) and mouse monoclonal antibodies specific for $\alpha$-actin $(1: 15,000$, Chemicon, Schwalbach, Germany) or GAPDH [1:10,000, Santa Cruz, (ORT) CA, USA].

Cellular fractionation. Generation of mitochondrial fractions was performed using the ApoAlert Cell Fractionation kit (Clontech, Mountain View, CA, USA, \#630105). After centrifugation and washing steps, the cell pellet was resuspended in ice-cold fractionation buffer mix containing protease inhibitors (Complete mini; Roche Molecular Biochemicals, Indianapolis, IN, USA) and $1 \mathrm{mM}$ 1,4-dithio-DL-threitol solution (DTT). After 10-min incubation on ice, cells were homogenized with a dounce tissue grinder. Efficiency of homogenization was controlled by microscopy. The homogenate was centrifuged at $700 \mathrm{x}$ g for $10 \mathrm{~min}$ at $4^{\circ} \mathrm{C}$ and the resulting supernatant at $10,000 \mathrm{x}$ g for $25 \mathrm{~min}$ at $4^{\circ} \mathrm{C}$. The pellet containing the mitochondrial fraction was resuspended in fractionation buffer mix and used for SDS polyacrylamide gel electrophoresis. 
Transfection with plasmid DNA. SW480A and Hct116 cells were seeded in 96 -well plates $\left(1,5 \times 10^{3}\right.$ per well) and incubated with lipofectamine (Invitrogen) and plasmids encoding for HMGB1 (pcDNA3-HMGB1-Flag; AG Roth, Institute of Pathology, University of Heidelberg, Heidelberg, Germany) and Bcl-2 (pcDNA3-Myc-Bcl-2; AG Reed, Burnham Institute for Medical Research, La Jolla, CA, USA) according to the manufacturer's instructions. The controls were transfected with the pcDNA3.1 neo plasmid (Invitrogen). For high expression levels transfected cells were used for experiments $24 \mathrm{~h}$ after incubation. For stable transfection with Bcl-2, cells were incubated with $1.4 \mathrm{mg} / \mathrm{ml}$ geneticin (Invitrogen, Auckland, The Netherlands) for 2-3 weeks. For generation of single clones, one cell per well was seeded in 96-well plates. These cells were cultivated for 4 weeks, expanded and after expression control in western blot analysis used for further experiments.

Coimmunoprecipitation. Stable SW480A and Hct116 Bcl-2 overexpressing transfectants were transiently transfected with pcDNA3-HMGB1-Flag as mentioned above. As control, the empty expression vector (neo) pcDNA3.1 neo was used. The cells were seeded in $10-\mathrm{cm}$ dishes $\left(6 \times 10^{6}\right.$ cells per dish) and adhered for $24 \mathrm{~h}$. Then, the cells were collected and resuspended in lysis buffer [ $30 \mathrm{mM}$ Tris (pH 7.4), $120 \mathrm{mM} \mathrm{NaCl}$, 2 mM EDTA, 2 mM KCl, 10\% glycerol, 1\% Triton X-100] containing a protease inhibitor mixture (Roche Molecular Biochemicals, Hannheim, Germany) and incubated for $30 \mathrm{~min}$ at $4^{\circ} \mathrm{C}$. After centrifugation for $30 \mathrm{~min}$ at $16,000 \mathrm{xg}$ and $4^{\circ} \mathrm{C}$, $50 \mu \mathrm{l}$ of the supernatant was frozen as lysate controls. The rest of the supernatant was incubated with $20 \mu \mathrm{l}$ M2-Flag beads (Sigma, Deisenhofen, Germany) at $4^{\circ} \mathrm{C}$ overnight. After extensive washing, immune complexes were fractionated by SDS polyacrylamide gel electrophoresis (PAGE) and transferred to nitrocellulose membranes for immunoblotting.

Precipitation of proteins. For precipitation of proteins in the supernatant, SW480A and Hct116 were seeded in 6-cm plates and adhered for $24 \mathrm{~h}$. Then, cells were treated with TRAIL $(500 \mathrm{ng} / \mathrm{ml})$ and/or ABT-737 $(100 \mu \mathrm{M})$ and in case of inhibition of endogenous HMGB1 additionally with glycyrrhizin (1 mM). The supernatant of these cells was centrifuged at $700 \mathrm{x} g$ and $4^{\circ} \mathrm{C}$. Supernatant $(200 \mu \mathrm{l})$ was mixed with $800 \mu \mathrm{l}$ methanol and $200 \mu \mathrm{l}$ chloroform (Fluka), mixed again, followed by the

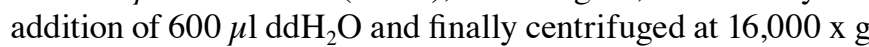
and $4^{\circ} \mathrm{C}$. The upper phase was removed. Methanol $(800 \mu \mathrm{l})$ was added to the other phase and centrifuged $2 \mathrm{~min}$ at $16,000 \mathrm{x} \mathrm{g}$ and $4^{\circ} \mathrm{C}$. The precipitated protein in the sediment was shortly dried and then mixed with lysis buffer.

FACS analysis. Apoptotic cells were assessed by flow cytometry with the PI-method. For this analysis, $1 \times 10^{6}$ SW480A and Hct116 colon carcinoma cells per sample were treated as indicated, washed, incubated with trypsin for $3 \mathrm{~min}$ at $37^{\circ} \mathrm{C}$, harvested, washed and stained with propidium iodine (Sigma-Aldrich). For detection of apoptotic cells a Galaxy proflow cytometer (Partec, Münster, Germany) equipped with a 488-nm air cooled argon laser and with filter combinations for propidium iodide and Flow Max software (Partec) was used. A total number of 10,000 cells were analyzed. After cell preparation according to Nicoletti et al (18) with modifications $(19,20)$ measurements were acquired in FI-3 in logarithmic mode and calculated by setting gates (RN-1) over the first three decades to detect apoptotic cells. Detection of vital (higher PI-intensity) and non-vital cells was assessed by PI-fluorescence signal.

For measurement of reactive oxygen species (ROS) $2 \times 10^{6}$ SW480A and Hct116 cells were seeded in 6-cm plates. Cells were treated with $80 \mathrm{nM}$ rhHMGB1 for $24 \mathrm{~h}$ and incubated with the fluorescent $\mathrm{H}_{2}$ DCF-DA (dichlorodihydrofluorescein diacetate, $10 \mu \mathrm{M}$, Invitrogen) for $1 \mathrm{~h}$ at $37^{\circ} \mathrm{C}$. The cells were trypsinized and centrifuged with $700 \mathrm{x} \mathrm{g}$ for $5 \mathrm{~min}$ at $4^{\circ} \mathrm{C}$. The pellet was washed twice with cold sterile Dulbecco's PBS containing $0.1 \%$ fetal calf serum and resuspended in $500 \mu 1$ sterile Dulbecco's PBS on ice. Oxidation of $\mathrm{H}_{2} \mathrm{DCF}$ to DCF by ROS and DiOC6 accumulation in mitochondria were measured immediately at FL-1 by flow cytometry using a FACSCalibur (Becton-Dickinson, Heidelberg, Germany).

Statistical analysis and determination of synergy. All data are means \pm SD of at least three independent replications performed in triplicate. Statistical significance was assessed by two-sided Student's t-test (Excel, Microsoft, Seattle, WA, USA). Values of $p<0.05$ were considered statistically significant. Synergy was evaluated by the fractional product method of Webb (21). In the fractional product method, the effect of two independently acting agents is defined as the product of the unaffected fractions after treatment with either agent alone: $f_{(1,2)}=f_{(1)} \times f_{(2)} / 100$. This formula allows the calculation of the predicted effect of co-treatment assuming that the two agents do not interact or cooperate in inducing their effects. If the relative percentage of surviving cells after co-treatment with the two drugs is below the calculated product $\mathrm{f}_{(1,2)}$, then the two drugs act in a synergistic manner. Results are considered significant if by Student's t-test p-values are $<0.05$ ( $\mathrm{p} p<0.05$, ${ }^{* *} \mathrm{p}<0.01,{ }^{* * *} \mathrm{p}<0.001$ in figures).

\section{Results}

HMGB1 induces a non-apoptotic type of cell death in colon carcinoma cells. In our earlier study we showed that in glioblastoma HMGB1 triggers a distinct type of cell death lacking features of classical apoptosis or necrosis (16). Interestingly, the response of epithelial cancer cells such as colon carcinoma cells seemed to substantially differ from the response of glioblastoma cells to HMGB1. Especially the metabolic response (e.g., ATP production) of CRC cells to HMGB1 was distinctly different (Gdynia et al, unpublished data). Therefore, we studied the cell death-related effects of HMGB1 on CRC cells in more detail. Firstly, we treated several CRC cell lines with recombinant HMGB1 and determined cellular survival after 3 days. Treatment with HMGB1 resulted in a strong inhibition of tumor cell proliferation and induction of cell death even at nanomolar HMGB1 concentrations (Fig. 1A). The HMGB1treated CRC cells exhibited distinct morphological changes in conventional phase contrast microscopy, namely the formation of large intracytoplasmic vacuoles (Fig. 1B, upper panel). In analogy to our findings in glioblastoma, these vacuoles corresponded to extremely enlarged mitochondria, so-called giant mitochondria, in electron microscopy (Fig. 1B, lower panel). To confirm that the observed changes are specifically caused by HMGB1 we co-treated the cells with glycyrrhizin, 
A

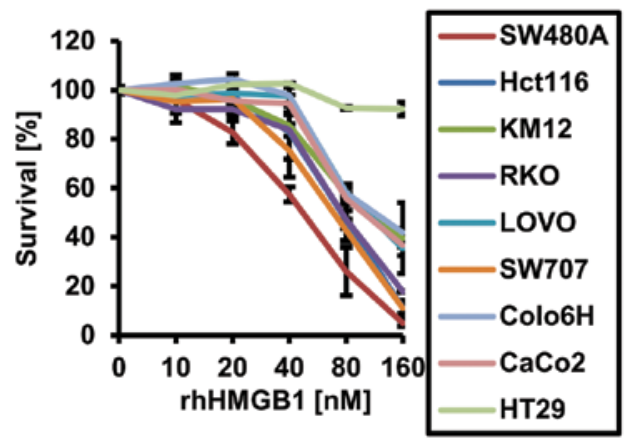

C

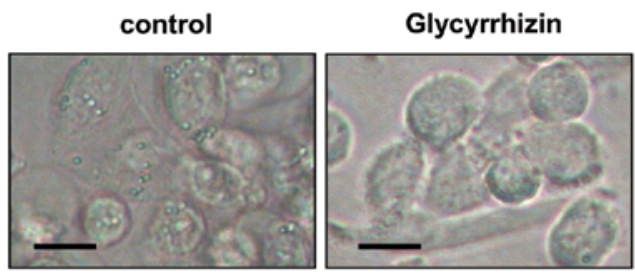

E

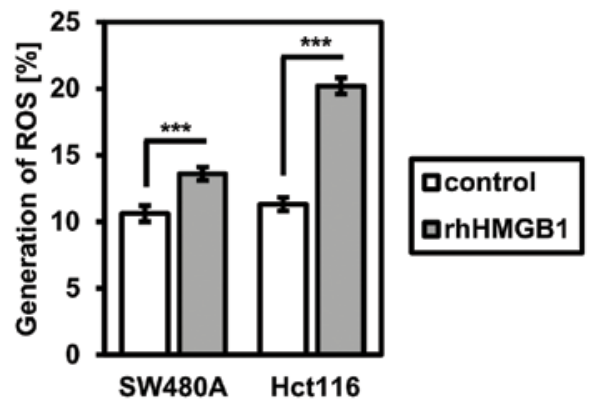

G
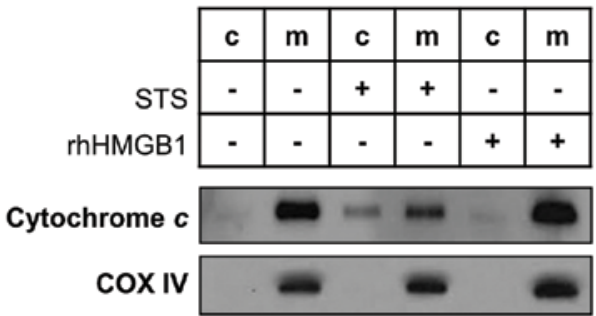

B
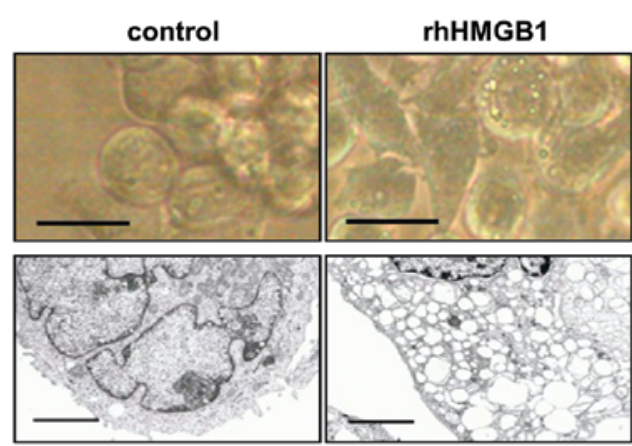

D

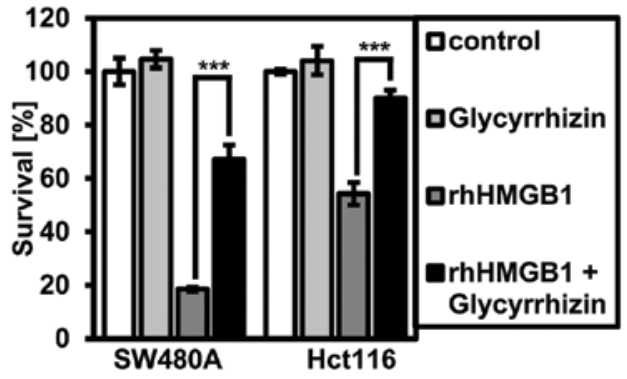

F

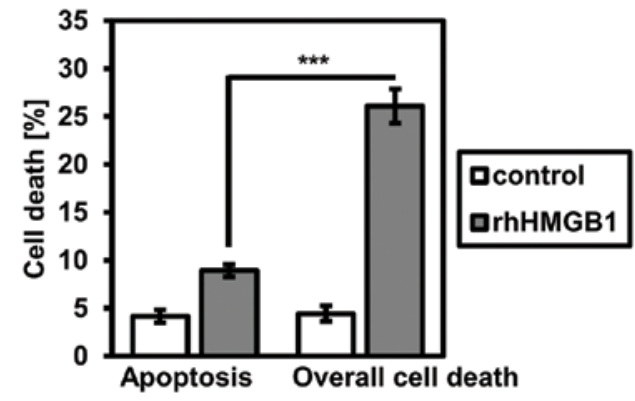

H

\begin{tabular}{r|c|c|c|c|c|}
\cline { 2 - 5 } TRAIL & - & ++ & + & - & + \\
\cline { 2 - 6 } & - & - & - & + & + \\
\cline { 2 - 6 } & &
\end{tabular}

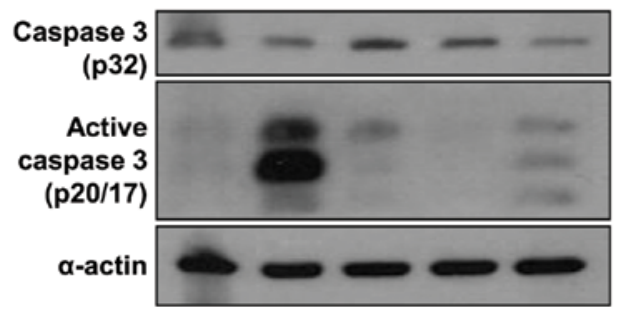

Figure 1. Cytotoxic effects of HMGB1 on colorectal carcinoma cells. (A) Nine colorectal carcinoma cell lines were treated with recombinant human HMGB1 (rhHMGB1) for $72 \mathrm{~h}$ at concentrations as indicated. Cellular survival was assessed by crystal violet staining ( $\mathrm{n}=3$, means $\pm \mathrm{SD}$ ). (B) Morphological changes of colorectal carcinoma cells after treatment with rhHMGB1 $(160 \mathrm{nM})$ over $24 \mathrm{~h}$. Upper panels, phase contrast microscopy of SW480A cells. Scale bars, $30 \mu \mathrm{m}$. Lower panels, electron microscopy of SW480A cells. Scale bars, $10 \mu \mathrm{m}$. In the control (left), the ultrastructure of mitochondria is maintained with numerous cristae and a dense matrix. After treatment with rhHMGB1 (right), formation of giant mitochondria can be observed. (C) Morphology of colorectal carcinoma cells after treatment with rhHMGB1 in presence or absence of the HMGB1 inhibitor glycyrrhizin. SW480A cells were treated with rhHMGB1 (80 nM) for $72 \mathrm{~h}$ and glycyrrhizin $(1 \mathrm{mM})$. Phase contrast microscopy; scale bars, $30 \mu \mathrm{m}$. (D) Protective effect of glycyrrhizin after HMGB1 treatment. SW480A and Hct116 colorectal carcinoma cells were treated with rhHMGB1 $(80 \mathrm{nM})$ for $72 \mathrm{~h}$ in presence or absence of glycyrrhizin $(1 \mathrm{mM})$. Cellular survival was assessed by crystal violet staining $\left(\mathrm{n}=3\right.$, means $\pm \mathrm{SD}$, Student's t-test, $\left.{ }^{* * *} \mathrm{p}<0.001\right)$. (E) ROS emission was measured after treatment with rhHMGB1 $(80 \mathrm{nM})$ for $24 \mathrm{~h}$ in SW480A and Hct116 cells by DCF fluorescence and fluorescence-activated cell sorting analysis ( $\mathrm{n}=3$, means \pm SD, Student's t-test, $\left.{ }^{* * *} \mathrm{p}<0.001\right)$. (F) Overall and apoptotic cell death after treatment with rhHMGB1. Apoptosis and overall cell death was measured after treatment of SW480A and Hct116 cells with rhHMGB1 $(80 \mathrm{nM}) 72 \mathrm{~h}$ by PI fluorescence and fluorescence-activated cell sorting according to the modified Nicoletti method (see Materials and methods) $\left(\mathrm{n}=3\right.$, means $\pm \mathrm{SD}$, Student's t-test, $\left.{ }^{* * * *} \mathrm{p}<0.001\right)$. Shown are the results in SW480A cells. (G) Release of cytochrome $c$ into the cytosolic fraction (c) was detected after treatment of cells with staurosporine (STS; $800 \mathrm{nM}, 6 \mathrm{~h}$ ), but not after treatment with rhHMGB1 $(80 \mathrm{nM}, 24 \mathrm{~h})$, although rhHMGB1 at this concentration induces substantial cell death after $72 \mathrm{~h} . \mathrm{m}$, mitochondrial fraction. COX IV serves as a mitochondrial loading control and control for successful fractionation. (H) Cleavage of caspase 3 is observed after TRAIL treatment, but not after rhHMGB1 treatment. SW480A colorectal carcinoma cells were treated with TRAIL $(+, 200 \mathrm{ng} / \mathrm{ml}$ over $24 \mathrm{~h} ;++, 500 \mathrm{ng} / \mathrm{ml}$ over $3 \mathrm{~h})$ and rhHMGB1 $(80 \mathrm{nM})$ for $24 \mathrm{~h}$. Similar results were observed also in Hct116 cells. 
A

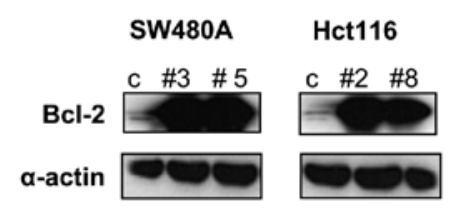

C

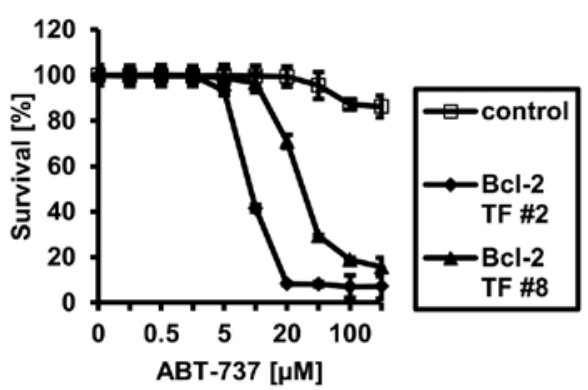

E

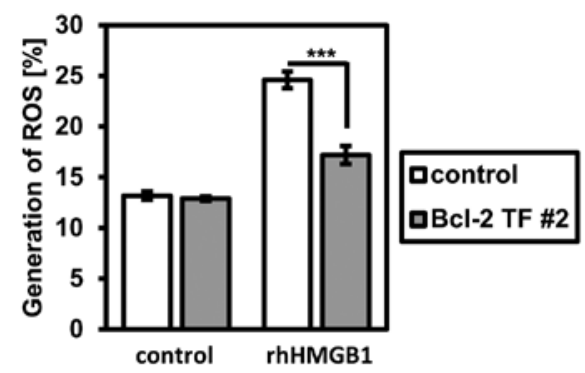

B

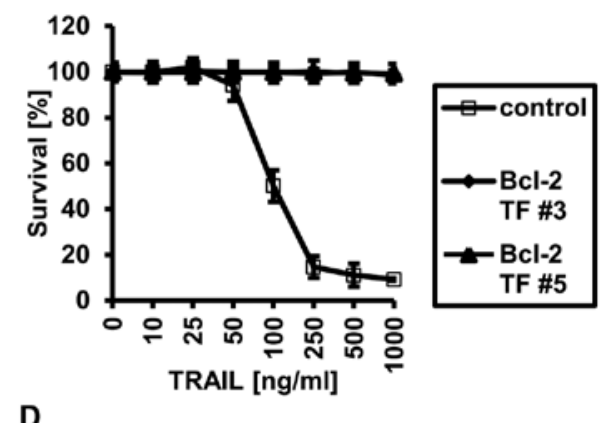

D

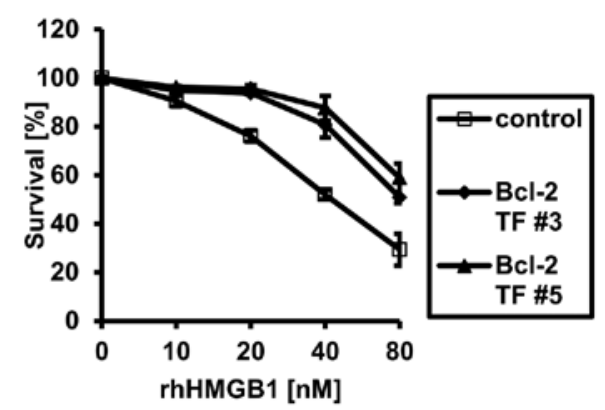

$\mathbf{F}$

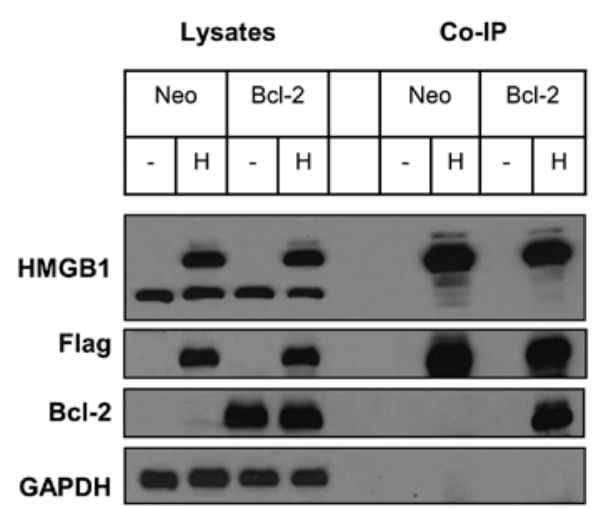

Figure 2. Bcl-2 protects CRC cells from HMGB1 induced cell death. (A) Stable overexpression of Bcl-2. Shown are the expression controls for Bcl-2 transfectants in SW480A and Hct116 colorectal cancer cells. Protein (35 $\mu \mathrm{g})$ was loaded per lane. $\alpha$-actin serves as loading control. (B-D) Effects of Bcl-2 overexpression on cytotoxicity induced by TRAIL (B), ABT-737 (C), or rhHMGB1 (D). SW480A and Hct116 Bcl-2 transfectants (TF) were treated with increasing concentrations of the compounds as indicated for $72 \mathrm{~h}$. Cellular survival was assessed by crystal violet staining ( $\mathrm{n}=3$, means \pm SD). (E) Reduction of ROS emission by Bcl-2 overexpression. ROS emission was measured after treatment with rhHMGB1 (80 nM) for 24 h in Hct116 Bcl-2 transfectants by DCF fluorescence and fluorescence-activated cell sorting analysis ( $\mathrm{n}=3$, means $\pm \mathrm{SD}$, Student's t-test, $\left.{ }^{* * *} \mathrm{p}<0.001\right)$. (F) SW480A Bcl-2 transfectants were transiently transfected with pcDNA3-HMGB1-Flag. Empty vector (pcDNA3.1) transfected cells served as negative control. Immunoprecipitation of Flag-HMGB1 was performed by using Flag binding beads. Immunoblot analysis was performed with anti-Bcl-2 antibody. -, control; H, HMGB1 transfected cells.

a tripertene which binds directly to HMGB1 and inhibits its extracellular cytokine function $(22,23)$. Both the formation of giant mitochondria (Fig. 1C) and the cytotoxic effect of HMGB1 (Fig. 1D) were efficiently blocked by glycyrrhizin. Since mitochondrial dysfunction is often associated with generation of reactive oxygen species (ROS) we tested whether HMGB1 alters the intracellular levels of ROS. A $\mathrm{H}_{2}$ DCF-DA FACS analysis demonstrated that HMGB1 induces a significant increase in ROS in CRC cells (Fig. 1E).

In contrast to the above mentioned cellular phenotype, the typical morphologic changes of apoptosis such as membrane blebbing, shrinking of the cell, fragmentation of the nucleus or the formation of apoptotic bodies were not observed. FACS analysis demonstrated only slight induction of apoptosis after HMGB1 treatment (Fig. 1F), and cytochrome $c$ release did not occur (Fig. 1G). Similarly, HMGB1 treatment did not result in cleavage of caspase 3 (Fig. $1 \mathrm{H})$. In contrast, treatment with the death ligand TRAIL strongly induced caspase 3 activation. Interestingly, caspase 3 cleavage was increased after combined treatment of cells with HMGB1 and TRAIL (Fig. 1H, right lane).

Bcl-2 protects $C R C$ cells from HMGB1-induced cytotoxicity. HMGB1 elicits in both glioblastoma cells (16) and in CRC cells (Fig. 1E) a significant increase in ROS. Therefore, we wondered whether the Bcl-2 protein, which stabilizes the outer mitochondrial membrane and prevents its permeabilization (24) would protect cells from HMGB1 cytotoxicity. To this end we created stably Bcl-2 overexpressing transfectants in the CRC cell lines SW480A and Hct116 (Fig. 2A). To confirm 
A
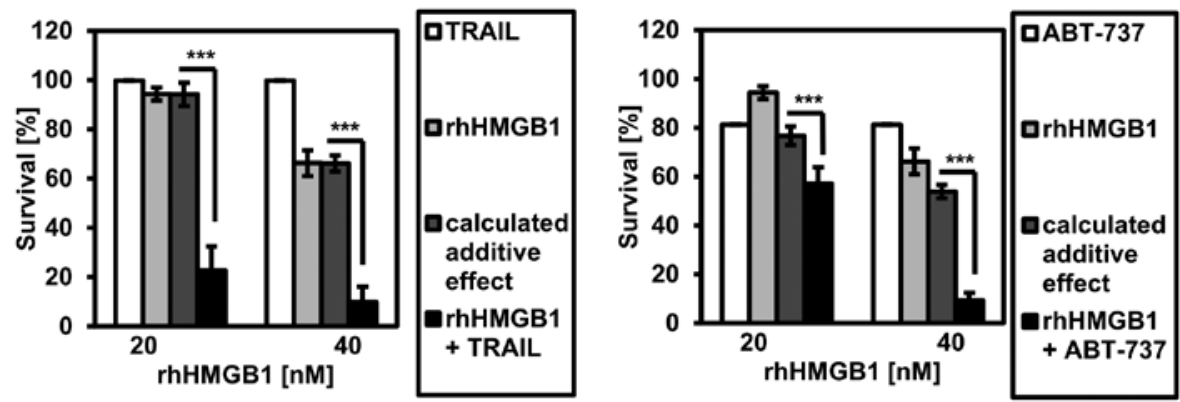

B

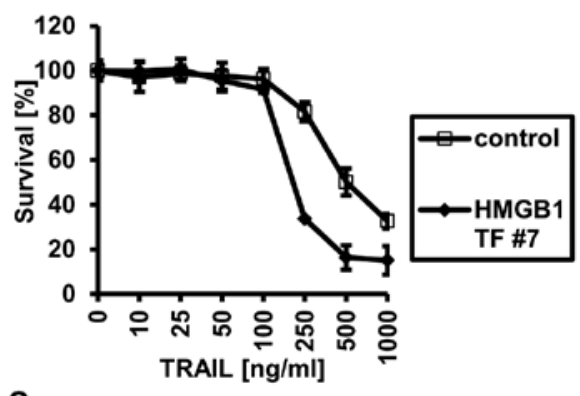

C

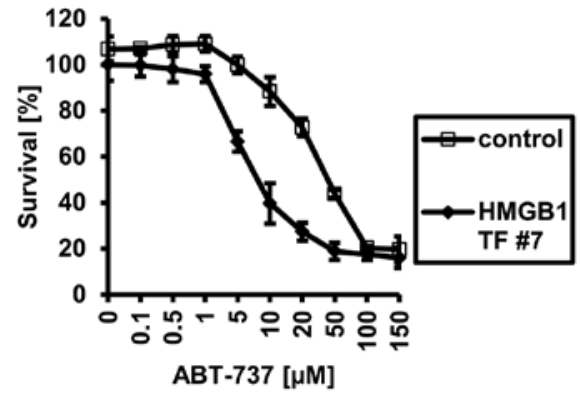

SW480A

Hct116
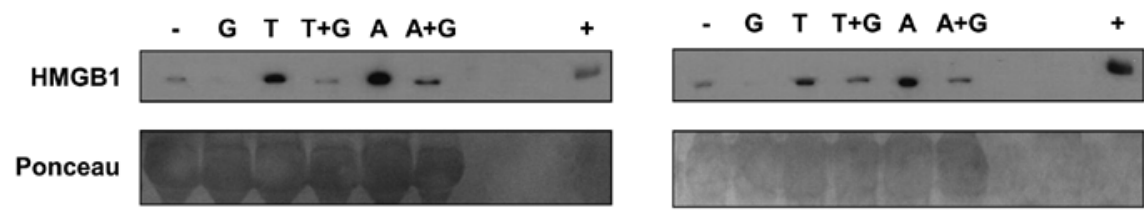

D
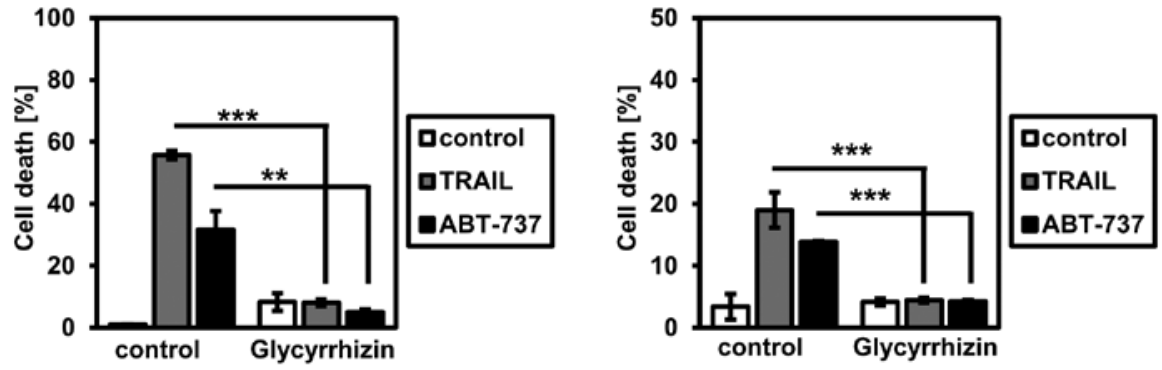

Figure 3. Synergistic interaction between HMGB1 and pro-apoptotic compounds. (A) SW480A colorectal cancer cells were treated with rhHMGB1 in combination with TRAIL $(50 \mathrm{ng} / \mathrm{ml})$ or ABT-737 $(1 \mu \mathrm{M})$. The cells were stained with crystal violet after $72 \mathrm{~h}$ and viability of the cells was determined. The column in dark grey shows the theoretically (calculated) surviving cell fraction after combined treatment in assumption that there is an additive effect (see Materials and methods) ( $\mathrm{n}=3$, means $\pm \mathrm{SD}$, Student's t-test, $\left.{ }^{* * *} \mathrm{p}<0.001\right)$. (B) Overexpression of HMGB1 sensitizes CRC cells for TRAIL and ABT-737. Stable SW480A HMGB1 transfectants (TF) were treated with increasing concentrations of TRAIL and ABT-737 as indicated for $24 \mathrm{~h}(\mathrm{n}=3$, means \pm SD). Cellular survival was assessed by crystal violet staining. (C) Release of endogenous HMGB1 into the cell culture supernatant after treatment with TRAIL or ABT-737. SW480A and Hct116 cells were treated with TRAIL $(500 \mathrm{ng} / \mathrm{ml})$ and ABT-737 $(100 \mu \mathrm{M})$ for $72 \mathrm{~h}$. For inhibition of endogenous HMGB1 glycyrrhizin $(1 \mathrm{mM})$ was used. As positive control served $0.1 \mu \mathrm{g}$ rhHMGB1. - , untreated; G, glycyrrhizin; T, TRAIL; T+G, TRAIL + glycyrrhizin; A, ABT-737; A+G, ABT-737 + glycyrrhizin; +, $0.1 \mu \mathrm{g}$ rhHMGB1. Ponceau staining of the membrane served as loading control. (D) Cell death after treatment with TRAIL or ABT-737 and inhibition by glycyrrhizin . Cell death was measured after treatment with TRAIL $(500 \mathrm{ng} / \mathrm{ml})$ or ABT-737 $(100 \mu \mathrm{M})$ in the presence or absence of glycyrrhizin $(1 \mathrm{mM})$ after $72 \mathrm{~h} \mathrm{by} \mathrm{PI}$ fluorescence and fluorescence-acivated cell sorting according to the modified Nicoletti method $(18-20)\left(n=3\right.$, means \pm SD, Student's t-test, $\left.{ }^{* * *} \mathrm{p}<0.001 ;{ }^{* *} \mathrm{p}<0.01\right)$.

the functionality of overexpressed $\mathrm{Bcl}-2$ we treated the cells with the death ligand TRAIL and the Bcl-2 family inhibitor ABT-737 (25). The Bcl-2 overexpressing CRC cells were completely resistant to the pro-apoptotic effects of TRAIL (Fig. 2B). In contrast, Bcl-2-transfected CRC cells turned out to be more susceptible to the cytotoxic activity of ABT-737 (Fig. 2C). This is in accordance with our earlier findings suggesting that a rapid release of pre-bound Bax from Bcl-2 can sensitize Bcl-2-overexpressing cells to ABT-737 (26). Next, we treated the CRC Bcl-2 transfectants with recombinant HMGB1 and observed a significant Bcl-2 dependent resistance (Fig. 2D). This resistance was paralleled by a substantially decreased generation of ROS in Bcl-2-overexpressing CRC cells (Fig. 2E). In an attempt to clarify how Bcl-2 can interfere 
A

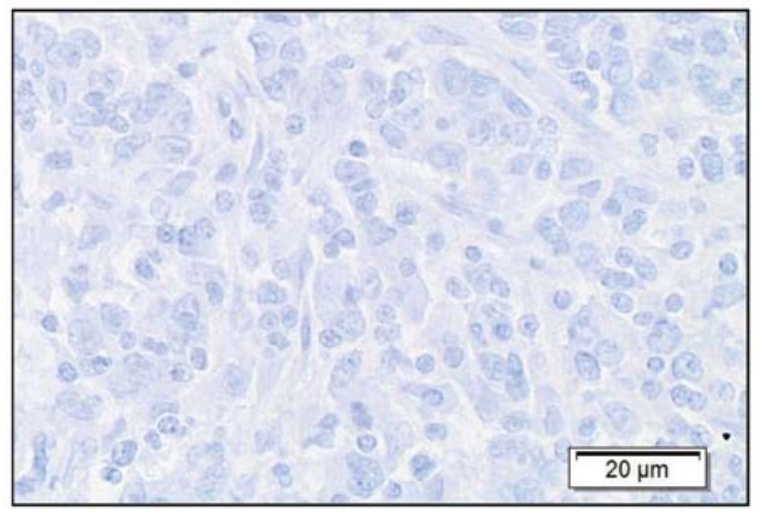

B

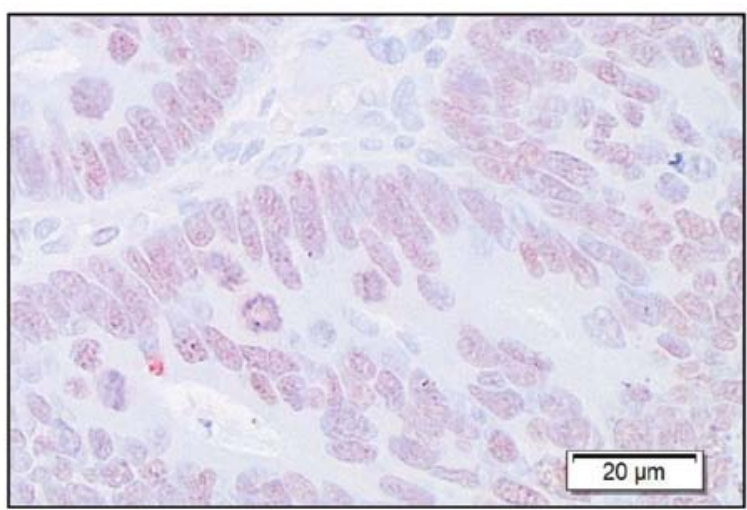

Figure 4. Expression of HMGB1 in human CRC tissue. CRC tissue samples were immunohistochemically analyzed for expression of HMGB1. (A) Example of loss of endogenous HMGB1 expression. (B) Retained expression of endogenous nuclear HMGB1. Bar, $20 \mu \mathrm{m}$. HMGB1 expression is visible as red-brown colour. Nuclei were lightly counter-stained by hematoxylin.

with HMGB1-induced ROS release we tested whether HMGB1 can physically interact with Bcl-2. A co-immunprecipitation experiment using transiently overexpressed HMGB1 in stably overexpressing $\mathrm{Bcl}-2$ cells suggested that both proteins bind to each other (Fig. 2F).

HMGB1 potentiates cell death induced by pro-apoptotic agents. In general, non-redundant cell death pathways can synergistically interact. Thus, we investigated the effects of a combined triggering of non-apoptotic and apoptotic cell death pathways by HMGB1 and TRAIL or ABT-737, respectively. Recombinant HMGB1 strongly potentiated TRAIL-induced cell death (Fig. 3A, left panel). Similarly, HMGB1 sensitized CRC cells to the cytotoxic effects of ABT-737 (Fig. 3A, right panel). A similar sensitizing effect was achieved when HMGB1 was endogenously overexpressed in CRC cells instead of incubating cells with recombinant HMGB1 (Fig. 3B). Next, we were interested whether TRAIL- or ABT-737-induced cell death by itself is associated with release of endogenous HMGB1. In fact, treatment of CRC cells with either TRAIL or ABT-737 induced a strong release of endogenous HMGB1 into the cell culture supernatant (Fig. 3C). The HMGB1 release could be blocked by the HMGB1 inhibitor glycyrrhizin. Consequently, we also tested whether this TRAIL- and ABT-737-dependent HMGB1 release contributes to the cytotoxic activity of these compounds. Importantly, the HMGB1 inhibitor glycyrrhizin was capable of substantially decreasing the cytotoxicity of TRAIL and ABT-737 (Fig. 3D), indicating that HMGB1 release contributes to the cytotoxic activity of these pro-apoptotic agents.

In vivo expression of $H M G B 1$ in human CRC. Given the cell death-regulating properties of HMGB1 we were interested in investigating the expression of HMGB1 in human CRC tissue samples and in correlating them with clinical data. Therefore, we examined the expression of HMGB1 immunohistochemically in a collection of 47 CRC samples, comprising CRC of pT stage $3(n=44)$ or pT stage $4(n=3)$, but differing in regard to their grade of malignancy $(\mathrm{G} 2$, moderately differentiated; $\mathrm{G} 3 / 4$, poorly differentiated) and the presence of lymph node metastasis (pN0, no lymph node metastasis; pN1+, with lymph node metastasis). Of 47 CRC samples, 26 showed absence or low levels of nuclear HMGB1 expression (Fig. 4A). Of these 26 cases, 21 carcinomas were poorly differentiated (G3/4) and/ or exhibited lymph node metastasis (pN1+) and/or showed blood vessel invasion (V1). In contrast, only 6 of 21 CRC with retained endogenous HMGB1 expression (Fig. 4B) showed these features of advanced, aggressive carcinomas (G3/4, pN1+, $\mathrm{V} 1)$, whereas the majority $(\mathrm{n}=15)$ of CRCs exhibited moderate differentiation (G2) and lack of lymph node metastasis (pN0) (sensitivity, 77.7\%; specificity, 75\%; positive predictive value, $81.48 \% ; \chi^{2}$ test: $\mathrm{t}=12.95 ; \mathrm{p}<0.005$, power $\left.>0.95\right)$. These data suggest that loss of HMGB1 expression is associated with a more aggressive phenotype and advanced (metastasized) disease in patients with CRC.

\section{Discussion}

HMGB1 is a multifunctional protein. As a danger protein, it plays an important role in innate immunity. Upon release, HMGB1 can exert autocrine or paracrine effects by triggering inflammation and activating the immune system $(8,13)$. However, high concentrations of HMGB1 are toxic and act as a classical mediator of septic shock. The molecular mechanisms of HMGB1-dependent toxicity are not well understood. In addition to its function as an immunologically active cytokine, HMGB1 is expressed as a nuclear 'architectural' transcription factor in most eukaryotic cells. HMGB1 is released into the extracellular space by necrotic cells, whereas in most apoptotic cells chromatin modifications lead to an intracellular retention of HMGB1 (8). Thus, the association of HMGB1 and regulation of cell death is intricate and might strictly depend on the cellular context and the microenvironment. Moreover, it is still unclear to which extent the HMGB1 protein is directly involved in the induction or modification of cell death.

In a recent report we described a novel distinct form of cell death in glioblastoma which is induced by HMGB1 (16). Similarly to glioblastoma cells, colorectal carcinoma cells exhibited the formation of giant mitochondria upon exposure to HMGB1, followed by rapid induction of cell death. This type of cell death significantly differs from the classical forms of cell death such as apoptosis. Specifically, HMGB1-induced 
cell death of CRC cells lacked caspase activation as well as the characteristic morphological features of apoptosis such as fragmentation of the nucleus, membrane blebbing, and apoptotic bodies $(27,28)$. Confirming the specificity of the observed HMGB1 effects, the cytotoxic activity of HMGB1 was strongly inhibited by glycyrrhizin. Glycyrrhizin is a natural anti-inflammatory and antiviral triterpene which inhibits the extracellular cytokine function of HMGB1 by direct binding $(23,29)$. In our model using recombinant HMGB1, the direct binding to glycyrrhizin might change the structure of HMGB1, so that the protein is not able to penetrate the cell membrane and translocate into the mitochondria, thereby preventing the formation of the giant mitochondria. Furthermore, glycyrrhizin has also effects on endogenous HMGB1, as we detected a decrease of released HMGB1 after treatment with TRAIL and ABT-737. This could be attributed to a degradation of endogenous HMGB1 or to an inhibition of its release. Moreover, glycyrrhizin inhibited not only HMGB1-induced cell death but also the formation of giant mitochondria. Most probably the mitochondrial effects of HMGB1 are decisive for HMGB1-triggered cytotoxicity (16), and our current data indicate that specific metabolic events targeting the cellular energy production by influencing oxidative phosphorylation and glycolysis mediate the cellular effects of HMGB1 (Gdynia et al, unpublished data).

Importantly, treatment of CRC cells with HMGB1 resulted in a substantial increase in intracellular ROS levels. Given the strong effect of HMGB1 on mitochondrial morphology (i.e., formation of giant mitochondria) we wondered whether mitochondrial membrane stability plays a role in the susceptibility to HMGB1. Since Bcl-2 regulates the cellular redox status and inhibits an excessive increase of ROS $(24,30,31)$, we tested the consequence of Bcl-2 overexpression in CRC cells. As a result, Bcl-2 significantly protected cells from HMGB1induced cell death and decreased the HMGB1-dependent increase in ROS production. Regarding the underlying mechanism, our findings suggest a physical interaction between Bcl-2 and HMGB1 which could negatively interfere with the ability of HMGB1 to exert its mitochondrial effects. However, further investigations have to clarify the precise mechanisms of this interaction.

Since Bcl-2 is one of the most prominent apoptosis-regulating proteins we wondered whether the distinct necrosis-like cell death induced by HMGB1 shows functional links with classical apoptosis when both signaling pathways are triggered. Interestingly, HMGB1-induced cell death and apoptosis triggered by the death ligand TRAIL or the Bcl-2/Bcl-XL family inhibitor ABT-737 exhibited a strong synergistic interaction. Of note, the amount of activated caspase 3 was increased in cells undergoing a combination treatment suggesting that HMGB1 sensitized CRC cells for the apoptotic signal transduction. Specifically, the HMGB1-triggered changes of mitochondrial structure and metabolic balance could prime cells to pro-apoptotic release of mitochondrial factors such as cytochrome $c$ or Smac. Vice versa, a priming of cells with activated apoptotic signaling to the HMGB1-induced cell death execution also appears possible. Especially events such as the ABT-737-dependent destabilization of mitochondrial membranes via permeabilization of the outer mitochondrial membrane might facilitate the translocation of HMGB1 to mitochondria and might potentiate generation of ROS. A similar mechanism may apply to the combination therapy with TRAIL that also elicits the activation of mitochondrial apoptosis in most cell types. Thus, the primary targeting of mitochondria through different routes by HMGB1 and proapoptotic agents could be mainly responsible for the observed synergistic induction of cell death. For other cytotoxic agents, synergistic toxicity with HMGB1 has been reported, such as for cisplatin; in this case HMGB1 supposedly shields the DNA from excision repair and thereby prevents the cell from cell cycle arrest leading to increased apoptosis $(32,33)$.

Another important finding of our study is the protective effect of glycyrrhizin on TRAIL- and ABT-737-induced cell death suggesting that HMGB1 also contributes to 'classical' apoptotic cell death. This hypothesis is in accordance with the above described HMGB1-dependent priming of cells to apoptosis. Alternatively, this effect could be caused by the mitochondrial membrane stabilizing effect of glycyrrhizin, which is known to exert antioxidant and anti-inflammatory effects $(34,35)$. Hence, glycyrrhizin suppresses oxidative stress-induced mitochondrial damage and exhibits a protective effect against oxidative cell injury (36), thereby possibly inhibiting the release of cytochrome $c$ into the cytosol and the activation of caspases.

Given the multiple functions of HMGB1 it is not surprising that there are contradictory findings regarding the relevance of HMGB1 expression levels in cancer tissue as assessed by immunohistochemistry. Numerous studies suggest that HMGB1 plays an important role in tumor growth and metastasis development, and thus links HMGB1 to poor prognosis in a variety of cancers such as prostate, breast, pancreas and colon (37-43). Regarding colorectal carcinomas the actual state of scientific knowledge is inconsistent. One study reported that HMGB1 expression was detected in most of the patients with Dukes' B or C, and that co-expression of RAGE and HMGB1 in these patients was associated with a significant worse prognosis (44). This hypothesis was also supported by the observation, that the expression of HMGB1 in the nucleus and the cytoplasm was inversely associated with the infiltration of T-cells and the prognosis of patients with stage IIIb CRCs (45). The immune response triggered by T-cells is believed to be important to limit early metastasis of CRC. It has been speculated that HMGB1 might modulate the immunologic response and contribute to the progression of CRC by promoting effective angiogenesis and immune escape. Another mechanism for the tumorigenic effect of HMGB1 in CRC could involve the upregulation of c-IAP2, an inhibitor of caspases and apoptosis, by enhancing NFKB activity (46). Furthermore, it has been reported, that HMGB1 can exert apoptotic effects on dendritic cells, which is associated with lymph node metastasis of CRC (47).

In contrast to this, there is also evidence for an antitumorigenic role of HMGB1 in CRC. It has been shown that HMGB1-induced TLR4 signaling is apparently necessary for an effective response to radiochemotherapy in tumor animal models (48). Furthermore, these findings are confirmed by the observation that patients with breast cancer carrying a loss of function TLR4 allele show a faster relapse after treatment with radiochemotherapy in comparison to patients with wild-type TLR4 alleles $(48,49)$. In addition to this, it has been 
reported, that the release of $\mathrm{HMGB} 1$ by $\mathrm{CRC}$ cells is required for an immunogenic cell death caused by oxaliplatin (50). Consistently patients with advanced stage CRC receiving an oxaliplatin-based chemotherapy, who carried a TLR4 loss-of-function allele, exhibited a reduced progression-free and overall survival, as compared with patients carrying the normal TLR4 allele.

Consistent with an antitumoral effect of HMGB1 our study shows that a high expression of HMGB1 significantly correlates with lower CRC stages. This observation is in agreement with the cytotoxic activity of HMGB1 demonstrated in our experiments, suggesting that carcinomas with increased HMGB1 expression could be more susceptible to induction of cell death and therefore exhibit a lower level of intrinsic resistance. Moreover, it is well known, that HMGB1 can act as a danger signaling protein. An overexpression of HMGB1 in carcinoma cells could lead to the induction of an immunological antitumoral host response thereby preventing fast progression of the tumor (48). The controversy between the results of our studies (high HMGB1 expression levels are significantly correlated with lower CRC stages) and the findings of the studies of Kuniyasu et al (44) (HMGB1 is associated with advanced-stage colon cancer) might be caused by different methods of investigations. In our study we used immunohistochemistry for the detection of HMGB1 expression whereas Kuniyasu et al performed an in situ hybridization for HMGB1 detection. By using this method Kuniyasu et al detected the HMGB1 expression on nucleic acid levels, while we demonstrated the protein expression in the tissue.

The findings of our above, and previous studies have clinical implications. Recombinant HMGB1 is highly cytotoxic to several types of cancer cells, e.g., colon carcinoma, glioblastoma, breast cancer and cervical carcinoma (16). At the same time, HMGB1 does not exert significant toxic effects on non-transformed cells, such as astrocytes or fibroblasts [(16) and data not shown]. In a glioblastoma mouse model, the systemic treatment with recombinant HMGB1 resulted in a strong reduction of tumor size without detectable side effects (16). The findings presented herein support a further evaluation of HMGB1 as a novel experimental treatment strategy for colon cancer. HMGB1 might overcome the intrinsic tumor cell resistance by triggering a distinct, non-apoptotic form of cell death. Especially in the case of apoptosis-resistant high-stage disease, this could represent a novel promising therapeutic approach. Moreover, both TRAIL and ABT-737 (or the derivatives thereof) are in clinical trials for patients with cancer (51-53). Regarding the well characterized problem of apoptosis-resistance leading to therapy-resistance, the HMGB1-dependent sensitization of CRC cells to apoptotic cell death could represent a promising novel approach to overcome the intrinsic cancer cell resistance. To this end, future investigations have to consider possible toxic side effects of HMGB1, especially pro-inflammatory reactions.

Our results show a high cytotoxic effect of HMGB1 in colorectal cancer by triggering a distinct non-apoptotic form of cell death. Both apoptosis inducing agents, TRAIL and ABT-737, which are used for clinical trials for patients with cancer, show significant synergistic effects with HMGB1, which could be used for an improved clinical therapy for patients with colorectal carcinomas.

\section{Acknowledgements}

We thank Hans-Walter Zentgraf $\dagger$ (German Cancer Research Center, Heidelberg, Germany) for performing the electron microscopy imaging; Sarah Messnard (University Hospital of Heidelberg) for immunohistochemical staining; Marcin Kaminski (German Cancer Research Center) for providing $\mathrm{H}_{2}$ DCF-DA; Tobias Kessler (German Cancer Research Center) for supporting the ROS-FACS analysis; Katrin Tagscherer (German Cancer Research Center) and Anne Fassl (German Cancer Research Center) for productive discussions; and Martina Keith (University Hospital of Heidelberg), Katarina Duglova (University Hospital of Heidelberg) and Maximilian Knoll (University Hospital of Heidelberg) for the excellent technical assistance.

\section{References}

1. Muller S, Scaffidi P, Degryse B, et al: New EMBO members' review: the double life of HMGB1 chromatin protein: architectural factor and extracellular signal. EMBO J 20: 4337-4340, 2001.

2. Lotze MT and Tracey KJ: High-mobility group box 1 protein (HMGB1): nuclear weapon in the immune arsenal. Nat Rev Immunol 5: 331-342, 2005.

3. Shirakawa H, Tsuda K and Yoshida M: Primary structure of nonhistone chromosomal protein HMG2 revealed by the nucleotide sequence. Biochemistry 29: 4419-4423, 1990.

4. Bustin M: Chromatin unfolding and activation by $\mathrm{HMGN}\left({ }^{*}\right)$ chromosomal proteins. Trends Biochem Sci 26: 431-437, 2001.

5. Wang H, Bloom O, Zhang M, et al: HMG-1 as a late mediator of endotoxin lethality in mice. Science 285: 248-251, 1999.

6. Andersson U, Wang H, Palmblad K, et al: High mobility group 1 protein (HMG-1) stimulates proinflammatory cytokine synthesis in human monocytes. J Exp Med 192: 565-570, 2000.

7. Zhang XD, Gillespie SK, Borrow JM and Hersey P: The histone deacetylase inhibitor suberic bishydroxamate regulates the expression of multiple apoptotic mediators and induces mitochondria-dependent apoptosis of melanoma cells. Mol Cancer Ther 3: 425-435, 2004.

8. Scaffidi P, Misteli T and Bianchi ME: Release of chromatin protein HMGB1 by necrotic cells triggers inflammation. Nature 418: 191-195, 2002.

9. Bell CW, Jiang W, Reich CF III and Pisetsky DS: The extracellular release of HMGB1 during apoptotic cell death. Am J Physiol Cell Physiol 291: C1318-C1325, 2006.

10. Wang H, Yang H, Czura CJ, Sama AE and Tracey KJ: HMGB1 as a late mediator of lethal systemic inflammation. Am J Respir Crit Care Med 164: 1768-1773, 2001.

11. Wang H, Ward MF and Sama AE: Novel HMGB1-inhibiting therapeutic agents for experimental sepsis. Shock 32: 348-357, 2009.

12. Yang H, Wang H, Czura CJ and Tracey KJ: The cytokine activity of HMGB1. J Leukoc Biol 78: 1-8, 2005.

13. Bianchi ME: HMGB1 loves company. J Leukoc Biol 86: 573-576, 2009.

14. Taniguchi N, Kawahara K, Yone K, et al: High mobility group box chromosomal protein 1 plays a role in the pathogenesis of rheumatoid arthritis as a novel cytokine. Arthritis Rheum 48: 971-981, 2003.

15. Dumitriu IE, Baruah P, Valentinis B, et al: Release of high mobility group box 1 by dendritic cells controls $T$ cell activation via the receptor for advanced glycation end products. J Immunol 174: 7506-7515, 2005.

16. Gdynia G, Keith M, Kopitz J, et al: Danger signaling protein HMGB1 induces a distinct form of cell death accompanied by formation of giant mitochondria. Cancer Res 70: 8558-8568, 2010.

17. Gillies RJ, Didier N and Denton M: Determination of cell number in monolayer cultures. Anal Biochem 159: 109-113, 1986.

18. Nicoletti I, Migliorati G, Pagliacci MC, Grignani F and Riccardi C: A rapid and simple method for measuring thymocyte apoptosis by propidium iodide staining and flow cytometry. J Immunol Methods 139: 271-279, 1991 
19. Ehemann V, Sykora J, Vera-Delgado J, Lange A and Otto HF: Flow cytometric detection of spontaneous apoptosis in human breast cancer using the TUNEL-technique. Cancer Lett 194: 125-131, 2003.

20. Ehemann V, Kern MA, Breinig M, et al: Establishment, characterization and drug sensitivity testing in primary cultures of human thymoma and thymic carcinoma. Int J Cancer 122 : 2719-2725, 2008.

21. Webb J: Effect of more than one inhibitor. Enzyme Metabolic Inhibitors 1: 488-512, 1963

22. Ulloa L, Ochani M, Yang H, et al: Ethyl pyruvate prevents lethality in mice with established lethal sepsis and systemic inflammation. Proc Natl Acad Sci USA 99: 12351-12356, 2002.

23. Mollica L, De Marchis F, Spitaleri A, et al: Glycyrrhizin binds to high-mobility group box 1 protein and inhibits its cytokine activities. Chem Biol 14: 431-441, 2007.

24. Krishna S, Low IC and Pervaiz S: Regulation of mitochondrial metabolism: yet another facet in the biology of the oncoprotein Bcl-2. Biochem J 435: 545-551, 2011.

25. Oltersdorf T, Elmore SW, Shoemaker AR, et al: An inhibitor of Bcl-2 family proteins induces regression of solid tumours. Nature 435: 677-681, 2005.

26. Tagscherer KE, Fassl A, Campos B, et al: Apoptosis-based treatment of glioblastomas with ABT-737, a novel small molecule inhibitor of Bcl-2 family proteins. Oncogene 27: 6646-6656, 2008.

27. Kroemer G, Galluzzi L, Vandenabeele P, et al: Classification of cell death: recommendations of the Nomenclature Committee on Cell Death 2009. Cell Death Differ 16: 3-11, 2009.

28. Kerr JF, Wyllie AH and Currie AR: Apoptosis: a basic biological phenomenon with wide-ranging implications in tissue kinetics. Br J Cancer 26: 239-257, 1972.

29. Girard JP: A direct inhibitor of HMGB1 cytokine. Chem Biol 14: 345-347, 2007

30. Chen ZX and Pervaiz S: Bcl-2 induces pro-oxidant state by engaging mitochondrial respiration in tumor cells. Cell Death Differ 14: 1617-1627, 2007.

31. Hockenbery DM, Oltvai ZN, Yin XM, Milliman CL and Korsmeyer SJ: Bcl-2 functions in an antioxidant pathway to prevent apoptosis. Cell 75: 241-251, 1993.

32. Huang JC, Zamble DB, Reardon JT, Lippard SJ and Sancar A HMG-domain proteins specifically inhibit the repair of the major DNA adduct of the anticancer drug cisplatin by human excision nuclease. Proc Natl Acad Sci USA 91: 10394-10398, 1994.

33. Wei M, Burenkova O and Lippard SJ: Cisplatin sensitivity in $\mathrm{Hmbg}^{1 /-}$ and $\mathrm{Hmbg} 1^{+/+}$mouse cells. J Biol Chem 278: 1769-1773, 2003.

34. Matsui S, Matsumoto H, Sonoda Y, et al: Glycyrrhizin and related compounds down-regulate production of inflammatory chemokines IL- 8 and eotaxin 1 in a human lung fibroblast cell line. Int Immunopharmacol 4: 1633-1644, 2004.

35. Nagai T, Egashira T, Yamanaka Y and Kohno M: The protective effect of glycyrrhizin against injury of the liver caused by ischemia-reperfusion. Arch Environ Contam Toxicol 20: 432-436, 1991.

36. Kim DE, Youn YC, Kim YK, Hong KM and Lee CS: Glycyrrhizin prevents 7-ketocholesterol toxicity against differentiated PC12 cells by suppressing mitochondrial membrane permeability change. Neurochem Res 34: 1433-1442, 2009.
37. Nestl A, Von Stein OD, Zatloukal K, et al: Gene expression patterns associated with the metastatic phenotype in rodent and human tumors. Cancer Res 61: 1569-1577, 2001.

38. Fedele M, Bandiera A, Chiappetta G, et al: Human colorectal carcinomas express high levels of high mobility group HMGI(Y) proteins. Cancer Res 56: 1896-1901, 1996.

39. Leman ES, Madigan MC, Brunagel G, Takaha N, Coffey DS and Getzenberg RH: Nuclear matrix localization of high mobility group protein $\mathrm{I}(\mathrm{Y})$ in a transgenic mouse model for prostate cancer. J Cell Biochem 88: 599-608, 2003.

40. Kuniyasu H, Chihara Y, Kondo H, Ohmori H and Ukai R: Amphoterin induction in prostatic stromal cells by androgen deprivation is associated with metastatic prostate cancer. Oncol Rep 10: 1863-1868, 2003

41. Ram TG, Reeves R and Hosick HL: Elevated high mobility group-I(Y) gene expression is associated with progressive transformation of mouse mammary epithelial cells. Cancer Res 53 2655-2660, 1993.

42. Bussemakers MJ, van de Ven WJ, Debruyne FM and Schalken JA: Identification of high mobility group protein I(Y) as potential progression marker for prostate cancer by differential hybridization analysis. Cancer Res 51: 606-611, 1991.

43. Dolde CE, Mukherjee M, Cho C and Resar LM: HMG-I/Y in human breast cancer cell lines. Breast Cancer Res Treat 71: 181-191, 2002.

44. Kuniyasu H, Sasaki T, Sasahira T, Ohmori H and Takahashi T: Depletion of tumor-infiltrating macrophages is associated with amphoterin expression in colon cancer. Pathobiology 71: 129-136, 2004.

45. Peng RQ, Wu XJ, Ding Y, et al: Co-expression of nuclear and cytoplasmic HMGB1 is inversely associated with infiltration of $\mathrm{CD} 45 \mathrm{RO}^{+} \mathrm{T}$ cells and prognosis in patients with stage IIIB colon cancer. BMC Cancer 10: 496, 2010.

46. Volp K, Brezniceanu ML, Bosser S, et al: Increased expression of high mobility group box 1 (HMGB1) is associated with an elevated level of the antiapoptotic c-IAP2 protein in human colon carcinomas. Gut 55: 234-242, 2006.

47. Kusume A, Sasahira T, Luo Y, et al: Suppression of dendritic cells by HMGB1 is associated with lymph node metastasis of human colon cancer. Pathobiology 76: 155-162, 2009.

48. Apetoh L, Ghiringhelli F, Tesniere A, et al: The interaction between HMGB1 and TLR4 dictates the outcome of anticancer chemotherapy and radiotherapy. Immunol Rev 220: 47-59, 2007.

49. Apetoh L, Ghiringhelli F, Tesniere A, et al: Toll-like receptor 4-dependent contribution of the immune system to anticancer chemotherapy and radiotherapy. Nat Med 13: 1050-1059, 2007.

50. Tesniere A, Schlemmer F, Boige V, et al: Immunogenic death of colon cancer cells treated with oxaliplatin. Oncogene 29: 482-491, 2010

51. Lim B, Scicchitano A, Beachler C, et al: FOLFIRI plus dulanermin (rhApo2L/TRAIL) in a patient with BRAF-mutant metastatic colon cancer. Cancer Biol Ther 14: 711-719, 2013.

52. Duiker EW, Mom CH, de Jong S, et al: The clinical trail of TRAIL. Eur J Cancer 42: 2233-2240, 2006.

53. Balakrishnan $\mathrm{K}$ and Gandhi V: Bcl-2 antagonists: a proof of concept for CLL therapy. Invest New Drugs 31: 1384-1394, 2013. 